\title{
USO DEL SISTEMA POPQ PARA LA CLASIFICACIÓN DE LOS PROLAPSOS: HOSPITAL PARROQUIAL DE SAN BERNARDO
}

\author{
Maritza Busquets C. ${ }^{1}$, Felipe Castillo F. a , Sebastián Bravo G. ${ }^{a}$, \\ Domingo Contreras $P$. $^{\text {a }}$ \\ 1 Unidad de Uroginecología, Departamento de Ginecología y Obstetricia, Universidad de los Andes, Hospital Parroquial \\ de San Bernardo.
}

a Internos, Facultad de Medicina, Universidad de los Andes.

\section{RESUMEN}

El sistema de Pelvic Organ Prolapse Quantification (POPQ) ha demostrado ser útil, fácil de aprender, rápido de realizar y con una buena confiabilidad intra e interobservador. Quisimos comenzar a aplicar este tipo de clasificación y compararla con la clasificación clásica de Baden y Walker descrita en la ficha clínica. Para esto se realizó un estudio observacional de 41 pacientes en que se comparó el sistema tradicional consignado en la ficha, con la medición de nueve puntos que utiliza el sistema POPQ. En 17\% de los casos ambos sistemas coincidieron en el diagnóstico del descenso de todos los elementos del prolapso: pared anterior, posterior y cuello uterino. El sistema POPQ permite un diagnóstico acabado del estado y magnitud del descenso de los órganos pelvianos.

\section{PALABRAS CLAVE: Prolapso genital, POPQ, sistema de clasificación, piso pélvico}

\section{SUMMARY}

The Pelvic Organ Prolapse Quantification (POPQ) System has prouved to be a good method for describing and quantifying pelvic organ prolapse. The objective of this observational study was to compare the classification consigned in the clinical records (Baden and Walker classification) with the new POPQ system. In the first 41 cases we proceed to evaluate our patients by mean of the POPQ system. In only $17 \%$ of the cases the two systems agreed in the diagnosis of the anterior, posterior and cervix descents. POPQ system allows an acute diagnosis of the state and magnitude of the descent of pelvic organs.

KEY WORDS: Pelvic organ prolapse, $P O P Q$, classification system, pelvic floor

\section{INTRODUCCIÓN}

Brubaker y Norton (1) presentaron los resultados de una revisión de más de 30 años en que analizaron sobre 100 artículos y 15 textos de estudio, no encontrando un consenso en relación a un sistema estándar de clasificación del prolapso de los órganos pelvianos. En 1996, un comité internacional publicó un documento proponiendo la utilización del "Pelvic Organ Prolapse Quantification system (POPQ)" (2). Este comité estuvo compuesto por miembros de la Sociedad Internacional de Continencia, la Sociedad Americana de Uroginecología y la Sociedad de Cirujanos Ginecológi- 
cos y actualmente este sistema estandarizado, está formalmente reconocido y adoptado por estas tres sociedades.

El sistema POPQ de clasificación del prolapso genital consiste en la medición de nueve puntos en vagina y perineo que se agrupan en una tabla de $3 \times 3$. Esto ya fue descrito en un artículo de revisión presentado en esta revista (3). La técnica ha demostrado ser útil, fácil de aprender, rápida de realizar y con una buena confiabilidad intra e interobservador $(4,5)$. Actualmente, aunque el sistema POPQ es el más utilizado en la literatura internacional, en más de la mitad de los estudios el sistema de clasificación no es citado o no está estandarizado (6).

El propósito de este estudio es analizar los cambios producidos por la aplicación de este nuevo método de clasificación, con respecto al diagnóstico de prolapso genital, comparándolo con el diagnóstico consignado previamente en la ficha, básicamente la clasificación de Baden y Walter (7). Este es un estudio preliminar, observacional, para evaluar la instauración definitiva de este sistema en el Servicio de Ginecología y Obstetricia del Hospital Parroquial de San Bernardo.

\section{PACIENTES Y MÉTODO}

Se midieron 41 pacientes entre 28 y 75 años (promedio 52 años) que consultaron en el Servicio de Ginecología y Obstetricia del Hospital Parroquial de San Bernardo, durante el año 2006. Como criterio de inclusión se eligieron las pacientes que ya habían sido evaluadas en un comité que revisa y decide las intervenciones, de manera que estaban clasificadas según el antiguo método de Baden y Walter (7). La mediana de la paridad vaginal (entre 1 y 11 partos) era 4 partos y el percentil 75 correspondió a 5 partos. Cuatro pacientes tenían el antecedente de histerectomía total. La clasificación de los prolapsos estaba hecha por los residentes de tercer año o los médicos tratantes.

Clásicamente, el método de Baden y Walter mide el descenso de los órganos pelvianos (histerocele, cistocele o rectocele) según se sitúan por debajo de las espinas ciáticas (primer grado), hasta nivel de introito (segundo grado) o por fuera del introito (tercer grado). La procidencia genital corresponde a la palpación del útero por fuera del introito. Se suele hablar de uretrocele o uretrocistocele cuando hay un descenso de la pared vaginal anterior a nivel de la uretra. Solemos hablar de prolapso completo cuando hay algún grado de histerocele (acompañado o no de cisto o rectocele), e incompleto cuando sólo hay descenso de alguna de las paredes vaginales.
Para la medición mediante el nuevo sistema $P O P Q$ utilizamos un pié de metro plástico (para poder ser esterilizado a gas) que consta de dos ramas horizontales entre las cuales se acomoda el objeto a medir y un vástago longitudinal cuyo extremo distal permite determinar una longitud determinada, un histerómetro y la media valva de un espéculo de tipo Collin. Se utilizó vaselina líquida para lubricar la vulva y las paredes vaginales; las pacientes fueron todas medidas por un mismo operador (MB) en posición de litotomía, con vejiga y recto vacíos, en estado de relajación y bajo el máximo pujo que pudiera efectuar la mujer. Se midió el descenso de las paredes vaginales y se clasificó el prolapso como de grado I, II, III o IV acompañado del punto de mayor descenso, según lo descrito en la técnica original y explicado por Braun y cols (3); se denominan con letra mayúscula los puntos medidos ( $A$ y B) y con letra minúscula si es pared anterior (a) o posterior ( $p)$. Los pasos que en general se siguieron fueron los siguientes:

1.Realización de especuloscopía y tacto vaginal bimanual de rutina con la paciente en posición de litotomía, vejiga y recto vacíos e impregnación con vaselina líquida de los genitales externos de la paciente para facilitar la inserción de las valvas.

2.Mediante tacto vaginal y con la mujer en posición relajada se introduce el histerómetro hasta el fondo de saco vaginal posterior, se mide el largo total vaginal y con pujo se mide la localización del Douglas (en referencia al himen o carúnculas himeneales). En caso de pacientes histerectomizadas este punto no se mide y se localiza el descenso, si lo hay, de la cicatriz en vez del cuello uterino (punto $\mathrm{C}$ ).

3.Mediante el tacto se identifica la porción más inferior del cuello uterino, lo que permite situar la punta del histerómetro, luego del pujo se mide su localización (siempre en referencia al himen o carúnculas himeneales); éste es el punto $\mathrm{Cx}$; se elimina el histerómetro.

4.Con el pié de metro, utilizando las ramas horizontales, se mide el hiato urogenital, desde el centro del meato uretral hasta el borde anterior del núcleo central del periné (que se identifica digitalmente mediante la palpación del elevador del ano y no por el repliegue de la horquilla), y luego el núcleo central del periné, desde este último punto identificado digitalmente hasta el centro del ano.

5.Utilizando la mitad del espéculo a modo de valva apoyado en la pared vaginal posterior, despejamos la pared vaginal anterior e identificamos con el vástago del pié de metro, el punto Aa $($ a $3 \mathrm{~cm}$ del meato uretral), luego, con pujo y utilizando también el vástago del pié de metro se lo ajusta de manera de medir la distancia entre el punto anteriormente 
identificado y el himen o las carúnculas himeneales. Acto seguido se determina si existe un punto entre éste y el cuello (o la cúpula) que descienda por debajo del anterior y se mide su localización con respecto al himen (Ba); si no lo hay, se localiza a igual distancia que el $\mathrm{Aa}$.

6.Se efectúa igual maniobra para la pared posterior, identificándose y localizando los puntos Ap y Bp.

7.Con la valva aún en la vagina y observando la pared vaginal posterior se realiza tacto rectal donde se evalúa la existencia de abombamientos en la zona alta y baja determinándose la presencia de un rectocele bajo o alto (se consignan de manera optativa según el sistema POPQ).

8.Durante este último tiempo se evalúa la presencia de enterocele. Esto lo hacemos tactando la valva con el dedo rectal y sintiendo si con el pujo se engruesa la pared vaginal por la presencia de intestino (enterocele por pulsión). El enterocele por tracción se determina según está establecido en el POPQ, cuando por el descenso del cuello se observa un abombamiento de la parte superior de la pared vaginal posterior, sin palparse intestino contra la valva que aún se mantiene en la vagina (en casos en que el cuello desciende por encima del introito, el espéculo de Collin permite identificar más fácilmente el enterocele por tracción).

9.Se miden los diámetros anterior y posterior entre las ramas horizontales del pié de metro, durante el pujo, tanto si había descenso de las paredes como si no (éstas son medidas optativas dentro del sistema POPQ).

Se transcribieron los nueve puntos estandarizados del sistema POPQ a una tabla de 3 por 3 casilleros y la presencia de enterocele por tracción o pulsión; además se consignaron los diámetros de las paredes anterior y posterior y la presencia o no de rectocele bajo y/o alto, que son optativos según el POPQ. Se efectuó una comparación caso a caso con la clasificación del prolapso que estaba previamente descrita en la ficha. El lapso entre una medición y la otra fue de un máximo de 6 meses. Si en la ficha no se consignaba la presencia de cistocele, rectocele o histerocele se consideró como la no existencia de tal descenso. Definimos como concordantes ambos sistemas cuando describían el mismo grado de severidad del prolapso, si por ejemplo, según el POPQ se clasificaba como etapa II debido a que el punto de mayor descenso se ubicaba a no más de $1 \mathrm{~cm}$ por fuera del introito, se consideró en concordancia con un prolapso de tercer grado de la antigua denominación (Figura 1). Se consideró como discordancia entre ambos tipos de clasificación, cuando no se traducían en el mismo grado de severidad del descenso o que algún tipo de descenso no estaba consignado en la ficha.

\section{CONCORDANCIA}

$1^{\circ}$ grado

por encima de 0 en POPQ

(est. I y II)

$2^{\circ}$ grado

en 0 de POPQ

(est. II)

$3^{\circ}$ grado

por debajo de cero en POPQ ( est. II, III, IV )

BASÁNDOSE EN PRIMERA INSTANCIA EN EL POPQ

$0=$ nivel de introito

Figura 1. Concordancia diagnóstica entre el sistema de Baden y Walter (7) y el sistema POPQ (2).

\section{RESULTADOS}

De los datos extraídos de las fichas se diagnosticaron 17 casos de prolapsos completos y 24 casos de prolapsos incompletos. Respecto de la clasificación mediante POPQ no hubo pacientes de estadios o grados 0 y l; 21 pacientes fueron catalogadas como prolapso grado II, 19 de grado III y 1 de grado IV.

Entre las pacientes con grado II, 14 de ellas presentaban el descenso más importante en la pared anterior (catalogadas como II Aa y/o Ba) y 6 presentaban un descenso de ambas paredes, de igual magnitud (descenso de Aa y/o Ba semejante al de Ap y/o Bp, clasificadas como llap); una paciente presentaba el descenso más importante constituido por el cuello (Ilcx), (Tabla I). De las 14 pacientes catalogadas como lla, 6 tenían además un descenso de la pared posterior (puntos Ap y/o Bp entre +1 y $-2 \mathrm{~cm}$.).

Respecto de la clasificación antigua, una paciente no tenía descrita la presencia de cistocele; en 4 pacientes cuyos cistoceles habían sido clasificados como de primer grado todas tenían el punto $\mathrm{Aa}$ o $\mathrm{Ba}$ a nivel 0 (a nivel de himen) y por ende no eran realmente de primer grado sino de segundo en la clasificación antigua; en 4 clasificadas correctamente como de tercer grado en ninguna protruía a más de $1 \mathrm{~cm}$ por fuera del himen. 
Tabla I

DISCORDANCIA CON RESPECTO A CLASIFICACIÓN CLÁSICA DE ESTADIOS II DE POPQ DEL DIAGNÓSTICO DE PARED ANTERIOR Y POSTERIOR

\begin{tabular}{|c|c|c|c|c|c|}
\hline \multirow[t]{2}{*}{$\mathrm{n}$} & \multirow{2}{*}{$\begin{array}{c}\text { Clasificación } \\
\text { POPQ }\end{array}$} & \multicolumn{2}{|c|}{ COINCIDE } & \multicolumn{2}{|c|}{ NO COINCIDE } \\
\hline & & $\begin{array}{l}\text { Pared } \\
\text { anterior }\end{array}$ & $\begin{array}{c}\text { Pared } \\
\text { posterior }\end{array}$ & $\begin{array}{l}\text { Pared } \\
\text { anterior }\end{array}$ & $\begin{array}{c}\text { Pared } \\
\text { posterior }\end{array}$ \\
\hline 14 & II $a^{1}$ & 9 & 5 & 5 & 9 \\
\hline 6 & II $a p^{2}$ & 5 & 2 & 1 & 4 \\
\hline 1 & $\| \mathrm{cx}$ & 1 & - & - & 1 \\
\hline 21 & - & 15 & 7 & 6 & 14 \\
\hline
\end{tabular}

Entre un total de 21 pacientes, en 15 el diagnóstico de pared anterior concuerda y en 14 el diagnóstico de la pared posterior no concuerda. En 16 pacientes que presentaban histerocele según $\mathrm{POPQ}$, sólo en 5 se había consignado en la ficha descenso del cuello uterino al pujo.

16 casos en que según POPQ existía además un descenso de pared posterior.

26 casos en que las paredes anterior y posterior descendían hasta introito (nivel 0).

En este grupo de 14 pacientes, 7 tenían descrito un rectocele de primero, segundo o tercer grado: según el POPQ en 4 de ellas no había descenso de los puntos Ap y/o Bp. Según la medición con $P O P Q$ de las 14, ocho no tenían descenso de pared posterior (Ap y Bp a -3) pero si se observó un rectocele bajo en 4 de ellas (optativo del POPQ) que habían sido clasificadas como rectoceles.

En los 6 casos en que existía un descenso semejante de las paredes anterior y posterior, en todos ellos, los puntos de mayor descenso de ambas paredes llegaban a nivel del himen; comparando con la clasificación antigua, todos los cistoceles, menos uno descrito como de nivel 1, estaban bien descritos de nivel 2. Respecto de la pared posterior en que según el POPQ todos llegaban a nivel del himen (IIABp) en tres casos la clasificación antigua detectó descensos por encima del himen (dos casos) o ausencia de rectocele (1 caso).

Por último, en este grupo de 21 pacientes catalogadas como grado II, 16 presentaban según el POPQ algún grado de descenso del cuello o cicatriz (el punto Cx o C respectivamente, está situado por debajo del largo total vaginal menos $2 \mathrm{~cm}$ ), y sólo 7 histeroceles estaban consignados en la ficha; 4 pacientes presentaban además un cierto grado de descenso del Douglas, pero en todos los casos el descenso era menor que el del cuello (el punto $D$ se localizó entre -5 y -8,5). En el caso del Ilcx, el histerocele estaba descrito como segundo grado, siendo que el cuello descendía a $1 \mathrm{~cm}$ por fuera del himen. No hubo casos de histeroceles descritos en la ficha que no estuvieran descritos por POPQ.

De las 19 pacientes con grado III, en 9 el descenso más importante estaba constituido por la pared anterior, en 5 por el cuello, en 4 pacientes por la pared posterior y en 1 caso por el Douglas (Tabla II).

Comparadas con la clasificación de la ficha, los nueve casos Illa con descensos importantes de pared anterior fueron descritos como cistocele de segundo grado en dos casos y el resto coincidía con un cistocele de tercer grado. De las 5 pacientes que poseían el descenso más importante de cuello, una (III Cx) había sido catalogada como histerocele de primer grado, el resto coincidía con un histerocele de tercer grado. En estos dos grupos (9 pacientes III a y 5 pacientes III Cx) se observó un descenso de la pared posterior según POPQ en 7 de ellas, de los cuales tres no estaban descritos en la ficha. En las otras 7 pacientes, no había descenso de la pared posterior (puntos Ap y Bp a -3), sin embargo, en 2 estaba descrito un rectocele de primer grado en la ficha. En aquellas 4 pacientes que presentaban el descenso más importante en pared posterior, 3 fueron catalogadas como rectoceles de primer y segundo grados, siendo que la pared descendía a más de $1 \mathrm{~cm}$ por fuera del introito; la cuarta fue correctamente evaluada y se corroboró un enterocele por pulsión descrito en la ficha, (en el sistema POPQ se adosa el adjetivo [pulsión] al lado del grado del prolapso: IIlp [pulsión]). De estas 4 pacientes, 3 habían sido sometidas a histerectomía total, y en ninguna de ellas estaba descrito un descenso 
Tabla II

DISCORDANCIA CON RESPECTO A CLASIFICACIÓN CLÁSICA DE ESTADIOS III Y IV DE POPQ DEL DIAGNÓSTICO DE PARED ANTERIOR Y POSTERIOR

\begin{tabular}{|c|c|c|c|c|c|}
\hline \multirow[t]{2}{*}{$\mathrm{n}$} & \multirow{2}{*}{$\begin{array}{c}\text { Clasificación } \\
\text { POPQ }\end{array}$} & \multicolumn{2}{|c|}{ COINCIDE } & \multicolumn{2}{|c|}{ NO COINCIDE } \\
\hline & & $\begin{array}{l}\text { Pared } \\
\text { anterior }\end{array}$ & $\begin{array}{c}\text { Pared } \\
\text { posterior }\end{array}$ & $\begin{array}{l}\text { Pared } \\
\text { anterior }\end{array}$ & $\begin{array}{c}\text { Pared } \\
\text { posterior }\end{array}$ \\
\hline 9 & III a & 7 & 4 & 2 & 5 \\
\hline $4^{1}$ & III $p$ & 0 & 2 & 4 & 2 \\
\hline 5 & III $c x^{2}$ & 2 & 3 & 3 & 2 \\
\hline 1 & III D & 1 & - & - & 1 \\
\hline 1 & IV c & - & - & 1 & 1 \\
\hline 20 & - & 10 & 9 & 10 & 11 \\
\hline
\end{tabular}

En estas pacientes en que el punto de mayor descenso protruía más allá del introito, en aproximadamente la mitad el diagnóstico de una de las paredes no coincidía.

1 tres pacientes histerectomizadas. ${ }^{2}$ cuello uterino.

de la cúpula; mediante el POPQ se identificó una de ellas con un descenso de $C$ (cicatriz) hasta el himen, que correspondía a la paciente catalogada como un grado III Bp [pulsión]. Hubo una segunda paciente con enterocele en esta serie, catalogada como grupo III a [pulsión] que se diagnosticó durante la evaluación con el POPQ.

En 8 de los nueve casos Illa se diagnosticó descenso de cuello según POPQ, en dos se diagnosticó además descenso del punto D que corresponde al Douglas. En 5 de éstas, el sistema tradicional no tenía consignado el prolapso como completo, es decir no había planteado el diagnóstico de histerocele (Tabla III). No hay casos en que se haya diagnosticado histerocele y que no se encontrara ningún grado de descenso del cuello en el POPQ. La paciente con grado IV, también histerectomizada, se trataba de un grado IV C (cicatriz) que coincidía con los diagnósticos consignados en la ficha.

\section{Tabla III}

\section{PRESENCIA O AUSENCIA DE HISTEROCELE SEGÚN SISTEMA DE CLASIFICACIÓN}

\begin{tabular}{cccc}
\hline Clasificación POPQ & $\begin{array}{c}\text { Casos con } \\
\text { descenso del cuello } \\
\text { o cúpula según } \\
\text { POPQ }\end{array}$ & $\begin{array}{c}\text { Consignado }{ }^{1} \text { en } \\
\text { ficha según Baden } \\
\text { \& Walker }\end{array}$ & No consignado \\
II (21) & 16 & 7 & 9 \\
III (19) & 15 & 10 & 5 \\
IV & 1 & 1 & 14 \\
TOTAL & 32 & 18 & 0 \\
\hline
\end{tabular}

\footnotetext{
${ }^{1}$ histerocele de cualquier grado (Baden Walker) consignado en la ficha, aunque no sea concordante con el grado de histerocele según POPQ.
} 
La concordancia entre ambos sistemas, según lo enunciado antes (Tabla IV), se puede resumir de la siguiente manera: en el diagnóstico exclusivo de pared anterior, ambos sistemas concuerdan en 21 (51\%) pacientes y de la pared posterior en 17 (41\%) pacientes. Respecto del histerocele o descenso de la cúpula en 21 pacientes la presencia, el grado de prolapso o su ausencia según POPQ, es concordante con lo descrito en la ficha $(51 \%)$.

\section{Tabla IV \\ CONCORDANCIA ENTRE AMBOS MÉTODOS EN LOS DISTINTOS ELEMENTOS COMPRO- METIDOS EN EL PROLAPSO}

\begin{tabular}{lc} 
Concordancia diagnóstica & $\mathrm{n}$ \\
\hline Pared anterior & $21(51 \%)$ \\
Pared posterior & $17(41 \%)$ \\
Histerocele & $18(44 \%)$ \\
Pared anterior y posterior & $11(27 \%)$ \\
Paredes e histerocele & $7(17 \%)$ \\
\hline
\end{tabular}

Si se compara la descripción del prolapso hecha mediante el POPQ con lo descrito en la ficha, respecto de la ausencia o el grado de descenso de ambas paredes (cistoceles y rectoceles), en 11 de $41(27 \%)$ hay coincidencia; si además agregamos el grado y la ausencia de histerocele, sólo en 7 pacientes (17\%) ambos sistemas coinciden.

Por último, respecto de los diámetros anterior y posterior, los diámetros de la pared anterior poseen una media más alta que los posteriores (en ambos grados II y III de descenso de la pared vaginal). En la pared vaginal anterior la media es de 3 a 3,5 para los grado II y de 5 en grados III. En la pared vaginal posterior, la media de 2 a 2,5 es igual para los grados II y III.

\section{DISCUSIÓN}

La distribución de los grados en nuestra serie obedece a que las pacientes están en espera de ser intervenidas. Las pacientes con POPQ de grado I o cistoceles y rectoceles de primer grado, no tienen en general indicación quirúrgica y en nuestro servicio, debido a la presión asistencial, se prioriza la paciente con prolapso más avanzado.

Respecto de la presencia o ausencia de un descenso de la pared anterior y posterior ambos siste- mas coinciden. Sin embargo, teniendo en cuenta que los cistoceles y/o rectoceles de primero, segundo y tercer grado de la clasificación antigua pueden resultar en un mismo grado II bajo el sistema POPQ, pudimos constatar que al medir cuantitativamente el prolapso no había equivalencia entre ambos sistemas. El diagnóstico en la ficha de severidad del descenso de pared anterior y posterior coincide con el POPQ en $51 \%$ y $41 \%$ respectivamente. En general, en el grupo grado II por POPQ había una tendencia a diagnosticar como de menor grado a los cistoceles que a los descensos reales de la pared anterior medidos por el POPQ (7 pacientes habían sido diagnosticadas como cistocele de primer grado y en realidad cualquiera de los puntos anteriores llegaba al himen). Esto puede deberse, a que el prolapso podría haber cambiado de etapa por el lapso de tiempo, o bien que la paciente en la medición del POPQ se comporte de una manera distinta que en un examen ginecológico. No creemos que un lapso de 6 meses entre ambas clasificaciones pueda influir significativamente en los resultados.

Durante la realización del POPQ se solicita a la paciente que realice maniobras de Valsalva en repetidas oportunidades, además como ya se describió, se utiliza vaselina líquida para facilitar la medición y disminuir la molestia de la introducción de la valva en la paciente, esto podría influir en que los descensos son más marcados que en el examen ginecológico de rutina. Esto podría explicar que en cuatro casos IIIp, tres fueran mal clasificados como de primer y segundo grados. Nos parece, sin embargo, que son los errores diagnósticos con la clasificación antigua lo que determina la gran diferencia entre ambos sistemas. Los diagnósticos consignados en la ficha están hechos por médicos ya sea residentes o médicos tratantes bastantes heterogéneos en su aproximación al prolapso genital.

En las pacientes cuyo descenso era de grado II o III de la pared anterior, los rectoceles descritos o no eran corroborados por el POPQ, o bien estaban clasificados según un descenso menor al real. En el grupo II, cuando el descenso más importante es de pared anterior, se describieron más rectoceles que los que había según POPQ. En el grado II cuando el descenso es anterior y posterior, en la mitad de los casos el rectocele descrito es menor a lo encontrado en el POPQ; algo semejante ocurre con aquellos casos Illp en que los rectoceles fueron también catalogados de menor grado según la antigua clasificación ( 3 de 4 casos). Cuando la pared anterior desciende considerablemente, 7 casos IIIa con descensos del punto Ap, sólo se describieron cuatro casos con rectocele según la clasificación antigua. Entre pacientes IIla y III Cx (14 casos), 
existía según POPQ 7 mujeres con descensos de pared posterior de los cuales sólo dos fueron correctamente identificados en la ficha. Es decir, que cuando la inspección se basa en el método tradicional, la presencia importante o más llamativa de un elemento nos hace evaluar con menos exactitud otro proceso concomitante disminuyendo nuestra atención sobre el resto del periné; por otra parte se puede suponer una tendencia a establecer arbitrariamente la presencia de rectocele cuando existe un cistocele.

Otro factor a tener en cuenta es que el abombamiento de la pared posterior vaginal puede constituir sólo un rectocele bajo y/o alto sin verdadero descenso del punto Ap, siendo catalogados como rectoceles de primer y segundo grados en la clasificación antigua. La presencia de desgarro vaginoperinal concomitante es frecuente en estos casos. La pared posterior parece ser más difícil de diagnosticar y de cuantificar siguiendo la clasificación clásica.

La pesquisa de histerocele fue deficientemente descrita por la clasificación antigua, ya que de 32 casos pesquisados por POPQ sólo 16, la mitad, habían sido descritos en la ficha. Con la clasificación antigua habría una tendencia a no diagnosticar los histeroceles y es quizás por esto que no es infrecuente que el diagnóstico se plantee durante la intervención y se decida en ese momento la realización de una histerectomía vaginal. Comparando los dos sistemas, si agregamos al diagnóstico de pared anterior y posterior, el diagnóstico correcto de histerocele, sólo en el $17 \%$ de los casos ambos sistemas coinciden. La utilización del POPQ obliga a reparar en todos los aspectos que pueden estar más o menos comprometidos.

Los diámetros transversales de la pared anterior son mayores que la pared posterior, nos parece que este hecho traduce el compromiso paravaginal de la fascia pubocervical en prolapsos de pared anterior.

En el sistema antiguo, la clasificación integra la presencia de histerocele, cistocele y rectocele, y con una ojeada podemos casi instantáneamente obtener un diagnóstico y sobre todo orientarnos sobre la conducta a seguir. Creemos que este sistema no debe abandonarse, puesto que siempre será necesario tener el concepto, por ejemplo de "histerocele" ya que sabemos que en los prolapsos completos de tercer grado se impone una histerectomía vaginal, que en otros estadios podría ser discutida. En el POPQ la clasificación del prolapso implica sólo el punto (o la pared) que presenta el descenso mayor; necesariamente hay que estudiar la tabla 3x3 para obtener la información global del estado perineal de la paciente. Para efectos de planificar la cirugía, por ejemplo, el primer sistema nos orienta más rápidamente sobre los pasos a seguir, sin embargo no cabe duda que la tabla $3 \times 3$ arroja mucho más información y más específica, ya que nos orienta sobre la cuantía de cada uno de los elementos que descienden con el pujo.

\section{CONCLUSIÓN}

El adoptar el sistema POPQ contribuye a realizar un acabado diagnóstico del estado del piso pelviano de la mujer y un método valedero para medir los resultados de las nuevas cirugías que se imponen actualmente en la literatura.

\section{BIBLIOGRAFÍA}

1. Brubaker L, Norton PI. Current clinical nomenclature for description of pelvic organ prolapse. J Pelvic Surg 1996;2:257-9.

2. Bump RC, Mattiasson A, Bok, et al. The standardization of terminology of female pelvic floor dysfunction. Am J Obstet Gynecol 1996;175:10-7.

3. Braun H, Rojas I, González F, et al. Prolapso genital severo: consideraciones clínicas, fisiopatológicas y de técnica quirúrgica al momento de su corrección. Rev Chil Obstet Ginecol 2004;69(2):149-56.

4. Hall AF, Theofrastous JP, Cundiff GC, et al. Interobserver and intraobserver reliability of the proposed International Continence Society, Society of Gynecologic Surgeons, and American Urogynecologic Society pelvic organ prolapse classification system. Am J Obstet Gynecol 1996;175:1467-71.

5. Kobak WH, Rosenberger K, Walters MD. Interobserver variation in the assessment of pelvic organ prolapse. Int Urogynecol J 1996;7:121-4.

6. Muir TW, Sep KJ, Barber MD. Adoption of the pelvis organ prolapse quantification system in peer-reviewed literature. Am J Obstet Gynecol 2003;189:1632-6.

7. Baden WF, Walker TA, Lindsay HJ. The vaginal profile. Tex Med J 1968;64:56-8. 